\title{
Linearizability Problem of Resonant Degenerate Singular Point for Polynomial Differential Systems
}

\author{
Yusen Wu, ${ }^{1}$ Cui Zhang, ${ }^{2}$ and Luju Liu ${ }^{1}$ \\ ${ }^{1}$ School of Mathematics and Statistics, Henan University of Science and Technology, Henan, \\ Luoyang 471003, China \\ ${ }^{2}$ College of Mathematics and Science, Luoyang Normal University, Henan, \\ Luoyang 471022, China
}

Correspondence should be addressed to Yusen Wu, wuyusen82714@yahoo.com.cn

Received 29 August 2011; Revised 14 January 2012; Accepted 27 January 2012

Academic Editor: Nicola Guglielmi

Copyright (C) 2012 Yusen Wu et al. This is an open access article distributed under the Creative Commons Attribution License, which permits unrestricted use, distribution, and reproduction in any medium, provided the original work is properly cited.

The linearizability (or isochronicity) problem is one of the open problems for polynomial differential systems which is far to be solved in general. A progressive way to find necessary conditions for linearizability is to compute period constants. In this paper, we are interested in the linearizability problem of $p:-q$ resonant degenerate singular point for polynomial differential systems. Firstly, we transform degenerate singular point into the origin via a homeomorphism. Moreover, we establish a new recursive algorithm to compute the so-called generalized period constants for the origin of the transformed system. Finally, to illustrate the effectiveness of our algorithm, we discuss the linearizability problems of $1:-1$ resonant degenerate singular point for a septic system. We stress that similar results are hardly seen in published literatures up till now. Our work is completely new and extends existing ones.

\section{Introduction}

In the qualitative theory of planar polynomial differential equations, the problem of characterizing isochronous centers has stimulated a great deal of effort but which is also remarkably intractable. The authors of [1] pointed out that "a center of an analytic system is isochronous if and only if there exists an analytic change of variables such that the original system is reduced to a linear system," thus an isochronous center is also called a linearizable center. A center is isochronous if the period of all period solutions in a neighborhood of it is constant. 
The problem of determining isochronous centers for polynomial vector fields with linear part of center type

$$
\begin{aligned}
& \frac{d x}{d t}=-y+\sum_{k=2}^{\infty} X_{k}(x, y), \\
& \frac{d y}{d t}=x+\sum_{k=2}^{\infty} Y_{k}(x, y),
\end{aligned}
$$

is the subject of much work, where

$$
X_{k}(x, y)=\sum_{\alpha+\beta=k} A_{\alpha \beta} x^{\alpha} y^{\beta}, \quad Y_{k}(x, y)=\sum_{\alpha+\beta=k} B_{\alpha \beta} x^{\alpha} y^{\beta}
$$

The representative results are quadratic isochronous centers, see [2]; isochronous centers of a linear center perturbed by third, fourth, and fifth degree homogeneous polynomials, see [3-5]; complex polynomial systems, see [1]; reversible systems, see [6, 7]; and isochronous centers of cubic systems with degenerate infinity, see $[8,9]$.

By means of complex transformation

$$
z=x+i y, \quad w=x-i y, \quad T=i t, \quad i=\sqrt{-1},
$$

system (1.1) becomes the following complex system:

$$
\begin{gathered}
\frac{d z}{d T}=z+\sum_{k=2}^{\infty} Z_{k}(z, w) \\
\frac{d w}{d T}=-w-\sum_{k=2}^{\infty} W_{k}(z, w),
\end{gathered}
$$

where

$$
\begin{aligned}
& Z_{k}(z, w)=\sum_{\alpha+\beta=k} a_{\alpha \beta} z^{\alpha} w^{\beta}=Y_{k}\left(\frac{z+w}{2}, \frac{z-w}{2 i}\right)-i X_{k}\left(\frac{z+w}{2}, \frac{z-w}{2 i}\right) \\
& W_{k}(z, w)=\sum_{\alpha+\beta=k} b_{\alpha \beta} w^{\alpha} z^{\beta}=Y_{k}\left(\frac{z+w}{2}, \frac{z-w}{2 i}\right)+i X_{k}\left(\frac{z+w}{2}, \frac{z-w}{2 i}\right) .
\end{aligned}
$$

Clearly, the coefficients of system (1.4) satisfy the conjugate condition, that is,

$$
\overline{a_{\alpha \beta}}=b_{\alpha \beta}, \quad \alpha \geq 0, \beta \geq 0, \alpha+\beta \geq 2 .
$$

As in [10], systems (1.1) and (1.4) are said to be concomitant. For isochronous center problem, we highlight the great contribution of Liu and Huang [11], which gave a new recursive algorithm to compute period constants at the origin of system (1.4). 
At times, the problem is restricted to the following polynomial differential system with linear part of $p:-q$ resonant saddle point type

$$
\frac{d x}{d t}=p x+P(x, y), \quad \frac{d y}{d t}=-q y+Q(x, y),
$$

where $p, q \in \mathbb{Z}^{+}, x, y, t \in \mathbb{R}, P$ and $Q$ are polynomials. After a time scaling $t \rightarrow p^{-1} t$, system (1.7) can be equivalently rewritten as

$$
\frac{d x}{d t}=x+P(x, y), \quad \frac{d y}{d t}=-\lambda y+Q(x, y),
$$

where $\lambda=q / p \in \mathbb{Q}^{+}$. As to the problems of linearizability of system (1.7), systematic research has been done. In [12], the necessary and sufficient conditions (15 cases) for linearizable systems of the $1:-2$ resonance were given. For the Lotka-Volterra system

$$
\dot{x}=x(1+a x+b y), \quad \dot{y}=y(-\lambda+c x+d y),
$$

necessary and sufficient conditions for linearizability in the case $\lambda \in \mathbb{N}$, that is the $1:-n$ resonant cases, were already known in [12]. In [13], some sufficient conditions were given in the case of general $\lambda$, and for the case $\lambda=p / 2$ or $2 / p, p \in \mathbb{N}^{+}$, necessary and sufficient conditions for linearizable systems were given. In [14], some new sufficient conditions for linearizable Lotka-Volterra system were presented. The linearizability was considered for $3:-4$ and $3:-5$ resonance in [15].

Wang and Liu [15] generalized the algorithm in [11]. Linearizability was investigated for the following general complex polynomial differential systems with a resonant singular point

$$
\begin{gathered}
\frac{d z}{d T}=z+\sum_{k=2}^{\infty} Z_{k}(z, w), \\
\frac{d w}{d T}=-\lambda w-\sum_{k=2}^{\infty} W_{k}(z, w) .
\end{gathered}
$$

Motivated by the above facts, in this paper, we concentrate on the linearizability problem for the polynomial differential system with a resonant degenerate singular point

$$
\begin{gathered}
\frac{d z}{d T}=p z^{n+1} w^{n}+\sum_{k=2 n+2}^{\infty} Z_{k}(z, w), \\
\frac{d w}{d T}=-q w^{n+1} z^{n}-\sum_{k=2 n+2}^{\infty} W_{k}(z, w),
\end{gathered}
$$

where $n \in \mathbb{N}, p, q \in \mathbb{Z}^{+},(p, q)=1, z, w, T$ are complex variables. 
Remark 1.1. (i) When $p=q$, without loss of generality, taking $p=q=1$, system (1.11) becomes

$$
\begin{gathered}
\frac{d z}{d T}=z^{n+1} w^{n}+\sum_{k=2 n+2}^{\infty} Z_{k}(z, w) \\
\frac{d w}{d T}=-w^{n+1} z^{n}-\sum_{k=2 n+2}^{\infty} W_{k}(z, w) .
\end{gathered}
$$

(ii) When $n=0$, system (1.11) becomes

$$
\begin{gathered}
\frac{d z}{d T}=p z+\sum_{k=2}^{\infty} Z_{k}(z, w), \\
\frac{d w}{d T}=-q w-\sum_{k=2}^{\infty} W_{k}(z, w),
\end{gathered}
$$

which is equivalent to system (1.10).

(iii) When $p=q=1, n=0$, system (1.11) becomes system (1.4).

Consequently, the proposed results are new and extend the existing ones with respect to systems (1.12) and (1.13).

The format of this paper is organized as follows. In Section 2, we recall some background definitions and lemmas which are very important in the proof of the main results in Section 3. In Section 3, we transfer degenerate singular point into the origin by a homeomorphic transformation and derive a new recursive algorithm for the calculation of generalized period constants at the origin of the transformed system. In Section 4, as an application of the new algorithm, we investigate the linearizability problem of $1:-1$ resonant degenerate singular point for a class of septic system. In the last section, we present the conclusions and remarks.

Most of the calculations in this paper have been done with the computer algebraic system-Mathematica.

\section{Preliminaries}

Lemma 2.1 (see $[12,13])$. System (1.13) is normalizable at the origin if and only if there exists an analytic change of variables:

$$
\xi=z+\Phi(z, w)=z+o(|z, w|), \quad \eta=w+\Psi(z, w)=w+o(|z, w|)
$$

bringing the system to its normal form

$$
\frac{d \xi}{d T}=p \xi\left(1+\sum_{i=1}^{\infty} p_{i} U^{i}\right), \quad \frac{d \eta}{d T}=-q \eta\left(1+\sum_{i=1}^{\infty} q_{i} U^{i}\right),
$$

where $U=\xi^{q} \eta^{p}$.

We write $\mu_{0}=\tau_{0}=0, \mu_{k}=p_{k}-q_{k}, \tau_{k}=p_{k}+q_{k}, k=1,2, \ldots$ 
Definition 2.2 (see [15]). For any positive integer $k, \mu_{k}$ is called the $k$ th singular point quantity of the origin of system (1.13). If system (1.13) is real planar differential system, $\mu_{k}$ is the $k$ th saddle quantity. If system (1.13) is the concomitant system of (1.1), $\mu_{k}$ is the $k$ th focus quantity. Moreover, the origin of system (1.13) is called a generalized center if $\mu_{k}=0, k=1,2, \ldots$

Definition 2.3 (see [15]). For any positive integer $k, \tau_{k}$ is called the $k$ th generalized period constant of the origin of system (1.13). And the origin of system (1.13) is called a generalized isochronous center if $\mu_{k}=\tau_{k}=0, k=1,2, \ldots$

Then from Lemma 2.1, and similar to the analytical procedure in [16], we have the following.

Lemma 2.4. System (1.13) is integrable at the origin if and only if one can derive uniquely the following formal series:

$$
\xi=z+\sum_{k+j=2}^{\infty} c_{k j} z^{k} w^{j}, \quad \eta=w+\sum_{k+j=2}^{\infty} d_{k j} w^{k} z^{j}
$$

where $c_{q i+1, p i}=d_{p i+1, q i}=0, i=1,2, \ldots$, such that

$$
\dot{\xi}=\xi h(\xi, \eta)=p \xi(1+O(|\xi, \eta|)), \quad \dot{\eta}=-q \eta h(\xi, \eta)=-q \eta(1+O(|\xi, \eta|))
$$

namely, $p_{i}=q_{i}(i=1,2, \ldots)$ in expression (2.2).

Lemma 2.5. System (1.13) is linearizable at the origin if and only if one can derive uniquely the following formal series (2.3), such that

$$
\dot{\xi}=p \xi, \quad \dot{\eta}=-q \eta,
$$

namely, $p_{i}=q_{i}=0(i=1,2, \ldots)$ in expression (2.2).

Lemma 2.6. System (1.13) is linearizable at the origin if and only if one can derive uniquely the change of coordinates (2.3) which linearizes the system, that is, $\mu_{k}=\tau_{k}=0, k=1,2, \ldots$.

Obviously, system (1.13) is linearizable at the origin if and only if the origin is a generalized isochronous center.

\section{Main Results}

Making the transformation

$$
z=z_{1}\left(z_{1} w_{1}\right)^{n+1}, \quad w=w_{1}\left(z_{1} w_{1}\right)^{n+1}, \quad d T=(2 n+3)\left(z_{1} w_{1}\right)^{-n(2 n+3)} d T_{1}
$$


and renaming $\left(z_{1}, w_{1}, T_{1}\right)$ by $(z, w, T)$, system (1.11) becomes

$$
\begin{aligned}
& \frac{d z}{d T}=p^{*} z+\sum_{\alpha+\beta=2 n+2}^{\infty}\left[(n+2) a_{\alpha \beta}+(n+1) b_{\beta+1, \alpha-1}\right] z^{\alpha+1} w^{\beta+1}(z w)^{(\alpha+\beta-2 n-2)(n+1)}, \\
& \frac{d w}{d T}=-q^{*} w-\sum_{\alpha+\beta=2 n+2}^{\infty}\left[(n+2) b_{\alpha \beta}+(n+1) a_{\beta+1, \alpha-1}\right] w^{\alpha+1} z^{\beta+1}(z w)^{(\alpha+\beta-2 n-2)(n+1)},
\end{aligned}
$$

where

$$
p^{*}=(n+1) q+(n+2) p, \quad q^{*}=(n+1) p+(n+2) q .
$$

Transformation (3.1) enables us to reduce the problem of $p:-q$ resonant degenerate singular point to that of the $p^{*}:-q^{*}$ resonant elementary origin.

Theorem 3.1. For system (3.2), one can derive uniquely the following formal series:

$$
f(z, w)=z+\sum_{k+j=2 n+4}^{\infty} c_{k j}^{\prime} z^{k} w^{j}, \quad g(z, w)=w+\sum_{k+j=2 n+4}^{\infty} d_{k j}^{\prime} w^{k} z^{j}
$$

where $c_{q^{*} i+1, p^{*} i}^{\prime}=d_{p^{*} i+1, q^{*} i}^{\prime}=0, i=1,2, \ldots$, such that

$$
\frac{d f}{d T}=p^{*} f(z, w)+\sum_{i=n+2}^{\infty} p^{*} p_{i}^{\prime} z^{q^{*} i+1} w^{p^{*} i}, \quad \frac{d g}{d T}=-q^{*} g(z, w)-\sum_{i=n+2}^{\infty} q^{*} q_{i}^{\prime} w^{p^{*} i+1} z^{q^{*} i},
$$

and when $p^{*} k-q^{*} j-p^{*} \neq 0$, (i.e., $\left.\left|k-q^{*} i-1\right|+\left|j-p^{*} i\right| \neq 0, i=0,1,2, \ldots\right), c_{k j}^{\prime}$ is determined by the following recursive formula:

$$
\begin{aligned}
& c_{k j}^{\prime}=\frac{1}{q^{*} j-p^{*}(k-1)} \\
& \times \sum_{\alpha+\beta=2 n+3}^{\infty}\left\{[(n+2)(k-\alpha)-(n+1)(j-\beta)-(\alpha+\beta-2 n-3)(n+1)] a_{\alpha, \beta-1}\right. \\
&\left.\quad-[(n+2)(j-\beta)-(n+1)(k-\alpha)-(\alpha+\beta-2 n-3)(n+1)] b_{\beta, \alpha-1}\right\} \\
& \quad \times c_{k-\alpha-(\alpha+\beta-2 n-3)(n+1), j-\beta-(\alpha+\beta-2 n-3)(n+1)^{\prime}}^{\prime}
\end{aligned}
$$


and when $q^{*} k-p^{*} j-q^{*} \neq 0$, (i.e., $\left.\left|k-p^{*} i-1\right|+\left|j-q^{*} i\right| \neq 0, i=0,1,2, \ldots\right), d_{k j}^{\prime}$ is determined by the following recursive formula:

$$
\begin{aligned}
& d_{k j}^{\prime}= \frac{1}{p^{*} j-q^{*}(k-1)} \\
& \times \sum_{\alpha+\beta=2 n+3}^{\infty}\left\{[(n+2)(k-\beta)-(n+1)(j-\alpha)-(\alpha+\beta-2 n-3)(n+1)] b_{\beta, \alpha-1}\right. \\
&\left.\quad-[(n+2)(j-\alpha)-(n+1)(k-\beta)-(\alpha+\beta-2 n-3)(n+1)] a_{\alpha, \beta-1}\right\} \\
& \quad \times d_{k-\beta-(\alpha+\beta-2 n-3)(n+1), j-\alpha-(\alpha+\beta-2 n-3)(n+1)}^{\prime} \\
&=\frac{1}{p^{*} j-q^{*}(k-1)} \\
& \quad \times \sum_{\alpha+\beta=2 n+3}^{\infty}\left\{[(n+2)(k-\alpha)-(n+1)(j-\beta)-(\alpha+\beta-2 n-3)(n+1)] b_{\alpha, \beta-1}\right. \\
&\left.\quad \quad-[(n+2)(j-\beta)-(n+1)(k-\alpha)-(\alpha+\beta-2 n-3)(n+1)] a_{\beta, \alpha-1}\right\} \\
& \quad \times d_{k-\alpha-(\alpha+\beta-2 n-3)(n+1), j-\beta-(\alpha+\beta-2 n-3)(n+1)^{\prime}}^{\prime}
\end{aligned}
$$

and for any positive integer $i, p_{i}^{\prime}$ and $q_{i}^{\prime}$ are determined by the following recursive formulae:

$$
\begin{aligned}
p_{i}^{\prime}=\frac{1}{p^{*}} \sum_{\alpha+\beta=2 n+3}^{\infty}\{ & {\left[(n+2)\left(q^{*} i+1-\alpha\right)-(n+1)\left(p^{*} i-\beta\right)-(\alpha+\beta-2 n-3)(n+1)\right] a_{\alpha, \beta-1} } \\
& \left.-\left[(n+2)\left(p^{*} i-\beta\right)-(n+1)\left(q^{*} i+1-\alpha\right)-(\alpha+\beta-2 n-3)(n+1)\right] b_{\beta, \alpha-1}\right\} \\
\times & c_{q^{*} i+1-\alpha-(\alpha+\beta-2 n-3)(n+1), p^{*} i-\beta-(\alpha+\beta-2 n-3)(n+1)^{\prime}}^{\prime}
\end{aligned}
$$

$$
\begin{aligned}
q_{i}^{\prime}=\frac{1}{q^{*}} \sum_{\alpha+\beta=2 n+3}^{\infty}\{ & {\left[(n+2)\left(p^{*} i+1-\beta\right)-(n+1)\left(q^{*} i-\alpha\right)-(\alpha+\beta-2 n-3)(n+1)\right] b_{\beta, \alpha-1} } \\
& \left.-\left[(n+2)\left(q^{*} i-\alpha\right)-(n+1)\left(p^{*} i+1-\beta\right)-(\alpha+\beta-2 n-3)(n+1)\right] a_{\alpha, \beta-1}\right\} \\
& \times d_{p^{*} i+1-\beta-(\alpha+\beta-2 n-3)(n+1), q^{*} i-\alpha-(\alpha+\beta-2 n-3)(n+1)}^{\prime} \\
=\frac{1}{q^{*}} \sum_{\alpha+\beta=2 n+3}^{\infty}\{ & \left\{\left[(n+2)\left(p^{*} i+1-\alpha\right)-(n+1)\left(q^{*} i-\beta\right)-(\alpha+\beta-2 n-3)(n+1)\right] b_{\alpha, \beta-1}\right. \\
& \left.-\left[(n+2)\left(q^{*} i-\beta\right)-(n+1)\left(p^{*} i+1-\alpha\right)-(\alpha+\beta-2 n-3)(n+1)\right] a_{\beta, \alpha-1}\right\} \\
\times & d_{p^{*} i+1-\alpha-(\alpha+\beta-2 n-3)(n+1), q^{*} i-\beta-(\alpha+\beta-2 n-3)(n+1)}^{\prime} .
\end{aligned}
$$


In expressions (3.6)-(3.9), for $1 \leq k+j \leq 2 n+3$, one has defined

$$
\begin{gathered}
c_{10}^{\prime}=1, \\
c_{k j}^{\prime}=0, \quad \text { for other }(k, j), \\
d_{10}^{\prime}=1, \\
d_{k j}^{\prime}=0, \quad \text { for other }(k, j),
\end{gathered}
$$

and if $k<0$ or $j<0$, let $a_{k j}=b_{k j}=c_{k j}^{\prime}=d_{k j}^{\prime}=0$.

Proof. Denote system (3.2) as

$$
\begin{aligned}
& \frac{d z}{d T}=p^{*} z+\sum_{\alpha+\beta=2 n+3}^{\infty}\left[(n+2) a_{\alpha, \beta-1}+(n+1) b_{\beta, \alpha-1}\right] z^{\alpha+1+(\alpha+\beta-2 n-3)(n+1)} w^{\beta+(\alpha+\beta-2 n-3)(n+1)}, \\
& \frac{d w}{d T}=-q^{*} w-\sum_{\alpha+\beta=2 n+3}^{\infty}\left[(n+2) b_{\beta, \alpha-1}+(n+1) a_{\alpha, \beta-1}\right] z^{\alpha+(\alpha+\beta-2 n-3)(n+1)} w^{\beta+1+(\alpha+\beta-2 n-3)(n+1)} .
\end{aligned}
$$

Differentiating $f(z, w)$ with respect to $T$ along the trajectories of system (3.2) yields

$$
\begin{aligned}
& \frac{d f}{d T}=\frac{d z}{d T}+\sum_{k+j=2 n+4}^{\infty} c_{k j}^{\prime}\left(k z^{k-1} w^{j} \frac{d z}{d T}+j z^{k} w^{j-1} \frac{d w}{d T}\right) \\
& =p^{*} z+\sum_{\alpha+\beta=2 n+3}^{\infty}\left[(n+2) a_{\alpha, \beta-1}+(n+1) b_{\beta, \alpha-1}\right] z^{\alpha+1+(\alpha+\beta-2 n-3)(n+1)} w^{\beta+(\alpha+\beta-2 n-3)(n+1)} \\
& +\sum_{k+j=2 n+4}^{\infty} c_{k j}^{\prime}\left\{k z ^ { k - 1 } w ^ { j } \left\{p^{*} z+\sum_{\alpha+\beta=2 n+3}^{\infty}\left[(n+2) a_{\alpha, \beta-1}+(n+1) b_{\beta, \alpha-1}\right]\right.\right. \\
& \left.\times z^{\alpha+1+(\alpha+\beta-2 n-3)(n+1)} w^{\beta+(\alpha+\beta-2 n-3)(n+1)}\right\} \\
& -j z^{k} w^{j-1}\left\{q^{*} w+\sum_{\alpha+\beta=2 n+3}^{\infty}\left[(n+2) b_{\beta, \alpha-1}+(n+1) a_{\alpha, \beta-1}\right]\right. \\
& \left.\left.\times z^{\alpha+(\alpha+\beta-2 n-3)(n+1)} w^{\beta+1+(\alpha+\beta-2 n-3)(n+1)}\right\}\right\}
\end{aligned}
$$


Journal of Applied Mathematics

$$
\begin{gathered}
=p^{*} z+p^{*} \sum_{k+j=2 n+4}^{\infty} c_{k j}^{\prime} z^{k} w^{j}+\sum_{k+j=2 n+4}^{\infty}\left(p^{*} k-q^{*} j-p^{*}\right) c_{k j}^{\prime} z^{k} w^{j} \\
+\sum_{\alpha+\beta=2 n+3}^{\infty}\left[(n+2) a_{\alpha, \beta-1}+(n+1) b_{\beta, \alpha-1}\right] z^{\alpha+1+(\alpha+\beta-2 n-3)(n+1)} w^{\beta+(\alpha+\beta-2 n-3)(n+1)} \\
+\sum_{k+j=2 n+4 \alpha+\beta=2 n+3}^{\infty} \sum_{k j}^{\infty}\left\{k\left[(n+2) a_{\alpha, \beta-1}+(n+1) b_{\beta, \alpha-1}\right]-j\left[(n+2) b_{\beta, \alpha-1}+(n+1) a_{\alpha, \beta-1}\right]\right\} \\
\times z^{k+\alpha+(\alpha+\beta-2 n-3)(n+1)} w^{j+\beta+(\alpha+\beta-2 n-3)(n+1)} .
\end{gathered}
$$

For $1 \leq k+j \leq 2 n+3$, let

$$
\begin{gathered}
c_{10}^{\prime}=1, \\
c_{k j}^{\prime}=0, \quad \text { for other }(k, j),
\end{gathered}
$$

then one arrives at

$$
\begin{gathered}
\sum_{\alpha+\beta=2 n+3}^{\infty}\left[(n+2) a_{\alpha, \beta-1}+(n+1) b_{\beta, \alpha-1}\right] z^{\alpha+1+(\alpha+\beta-2 n-3)(n+1)} w^{\beta+(\alpha+\beta-2 n-3)(n+1)} \\
=\sum_{k+j=1}^{2 n+3} \sum_{\alpha+\beta=2 n+3}^{\infty} c_{k j}^{\prime}\left\{k\left[(n+2) a_{\alpha, \beta-1}+(n+1) b_{\beta, \alpha-1}\right]-j\left[(n+2) b_{\beta, \alpha-1}+(n+1) a_{\alpha, \beta-1}\right]\right\} \\
\times z^{k+\alpha+(\alpha+\beta-2 n-3)(n+1)} w^{j+\beta+(\alpha+\beta-2 n-3)(n+1)},
\end{gathered}
$$

thus

$$
\begin{aligned}
\frac{d f}{d T}= & p^{*} f(z, w)+\sum_{k+j=2 n+4}^{\infty}\left(p^{*} k-q^{*} j-p^{*}\right) c_{k j}^{\prime} z^{k} w^{j} \\
+ & \sum_{k+j=1}^{\infty} \sum_{\alpha+\beta=2 n+3}^{\infty}\left\{[(n+2) k-(n+1) j] a_{\alpha, \beta-1}-[(n+2) j-(n+1) k] b_{\beta, \alpha-1}\right\} \\
& \times c_{k j}^{\prime} z^{k+\alpha+(\alpha+\beta-2 n-3)(n+1)} w^{j+\beta+(\alpha+\beta-2 n-3)(n+1)} .
\end{aligned}
$$


Subscript transformation $k^{\prime}=k+\alpha+(\alpha+\beta-2 n-3)(n+1), j^{\prime}=j+\beta+(\alpha+\beta-2 n-3)(n+1)$ implies $k^{\prime}+j^{\prime}=k+j+(\alpha+\beta-2 n-2)(2 n+3) \geq 2 n+4$. Still denoting $\left(k^{\prime}, j^{\prime}\right)$ with $(k, j)$, then one derives

$$
\begin{gathered}
\sum_{k+j=1}^{\infty} \sum_{\alpha+\beta=2 n+3}^{\infty}\left\{[(n+2) k-(n+1) j] a_{\alpha, \beta-1}-[(n+2) j-(n+1) k] b_{\beta, \alpha-1}\right\} \\
\times c_{k j}^{\prime} z^{k+\alpha+(\alpha+\beta-2 n-3)(n+1)} w^{j+\beta+(\alpha+\beta-2 n-3)(n+1)} \\
=\sum_{k+j=2 n+4}^{\infty} \sum_{\alpha+\beta=2 n+3}^{\infty}\left\{[(n+2)(k-\alpha)-(n+1)(j-\beta)-(\alpha+\beta-2 n-3)(n+1)] a_{\alpha, \beta-1}\right. \\
\left.\quad-[(n+2)(j-\beta)-(n+1)(k-\alpha)-(\alpha+\beta-2 n-3)(n+1)] b_{\beta, \alpha-1}\right\} \\
\times c_{k-\alpha-(\alpha+\beta-2 n-3)(n+1), j-\beta-(\alpha+\beta-2 n-3)(n+1)}^{\prime} z^{k} w^{j} .
\end{gathered}
$$

Therefore,

$$
\begin{gathered}
\frac{d f}{d T}=p^{*} f(z, w) \\
+\sum_{k+j=2 n+4}^{\infty}\left\{\left(p^{*} k-q^{*} j-p^{*}\right) c_{k j}^{\prime}\right. \\
+\sum_{\alpha+\beta=2 n+3}^{\infty}\left\{[(n+2)(k-\alpha)-(n+1)(j-\beta)-(\alpha+\beta-2 n-3)(n+1)] a_{\alpha, \beta-1}\right. \\
\left.-[(n+2)(j-\beta)-(n+1)(k-\alpha)-(\alpha+\beta-2 n-3)(n+1)] b_{\beta, \alpha-1}\right\} \\
\left.\times c_{k-\alpha-(\alpha+\beta-2 n-3)(n+1), j-\beta-(\alpha+\beta-2 n-3)(n+1)}^{\prime}\right\} z^{k} w^{j} .
\end{gathered}
$$

Denote that

$$
\begin{aligned}
h_{k j}=\left(p^{*} k-q^{*} j-p^{*}\right) c_{k j}^{\prime} \\
+\sum_{\alpha+\beta=2 n+3}^{\infty}\left\{[(n+2)(k-\alpha)-(n+1)(j-\beta)-(\alpha+\beta-2 n-3)(n+1)] a_{\alpha, \beta-1}\right. \\
\left.\quad-[(n+2)(j-\beta)-(n+1)(k-\alpha)-(\alpha+\beta-2 n-3)(n+1)] b_{\beta, \alpha-1}\right\} \\
\times c_{k-\alpha-(\alpha+\beta-2 n-3)(n+1), j-\beta-(\alpha+\beta-2 n-3)(n+1)^{\prime}}^{\prime}
\end{aligned}
$$

When $p^{*} k-q^{*} j-p^{*} \neq 0$, take $h_{k j}=0$, else take $h_{k j}=p^{*} p_{i}^{\prime}\left(k=q^{*} i+1, j=p^{*} i, i=1,2, \ldots\right)$, then one obtains

$$
\frac{d f}{d T}=p^{*} f(z, w)+\sum_{i=n+2}^{\infty} p^{*} p_{i}^{\prime} z^{* i+1} w^{p^{*} i}
$$


When $p^{*} k-q^{*} j-p^{*} \neq 0$, from $h_{k j}=0$, one gets (3.6); and when $p^{*} k-q^{*} j-p^{*}=0$, from $h_{k j}=h_{q^{*} i+1, p^{*} i}=p_{i}^{\prime}$, one gets (3.8).

For $g(z, w)$, in the same way as $f(z, w)$ was done, one can get (3.7) and (3.9).

The relations between $p_{i}, q_{i}$ and $p_{i}^{\prime}, q_{i}^{\prime}(i=1,2, \ldots)$ are as follows.

Theorem 3.2. Let $p_{0}=q_{0}=p_{0}^{\prime}=q_{0}^{\prime}=0$. If there exists a positive integer $l$, such that

$$
p_{0}=q_{0}=p_{1}=q_{1}=\cdots=p_{l-1}=q_{l-1}=0,
$$

then

$$
p_{0}^{\prime}=q_{0}^{\prime}=p_{1}^{\prime}=q_{1}^{\prime}=p_{l-1}^{\prime}=q_{l-1}^{\prime}=0, \quad p_{l}=p_{l}^{\prime}, \quad q_{l}=q_{l}^{\prime}
$$

per contra holds as well.

Proof. Let $l$ be any a positive integer, and

$$
\begin{array}{ll}
\tilde{\varphi}(z, w)=z+\sum_{k+j=2 n+4}^{\left(p^{*}+q^{*}\right) l+1} c_{k j} z^{k} w^{j}, & \tilde{\psi}(z, w)=w+\sum_{k+j=2 n+4}^{\left(p^{*}+q^{*}\right) l+1} d_{k j} w^{k} z^{j}, \\
\tilde{f}(z, w)=z+\sum_{k+j=2 n+4}^{\left(p^{*}+q^{*}\right) l+1} c_{k j}^{\prime} z^{k} w^{j}, & \tilde{g}(z, w)=w+\sum_{k+j=2 n+4}^{\left(p^{*}+q^{*}\right) l+1} d_{k j}^{\prime} w^{k} z^{j} .
\end{array}
$$

When expression (3.20) holds, from (2.3) we have

$$
\frac{d \tilde{\varphi}}{d T}=p^{*} \tilde{\varphi}+p^{*} p_{l} z^{q^{*} l+1} w^{p^{*} l}+\text { h.o.t., } \quad \frac{d \widetilde{\psi}}{d T}=-q^{*} \widetilde{\psi}-q^{*} q_{l} w^{p^{*} l+1} z^{q^{* l} l}+\text { h.o.t. },
$$

where h.o.t. stands for higher order term.

From expression (3.5), we get

$$
\frac{d \tilde{f}}{d T}=p^{*} \tilde{f}+\sum_{i=n+2}^{l} p^{*} p_{i}^{\prime} z^{*} z^{*+1} w^{p^{*} i}+\text { h.o.t., } \quad \frac{d \tilde{g}}{d T}=-q^{*} \tilde{g}-\sum_{i=n+2}^{l} q^{*} q_{i}^{\prime} w^{p^{*} i+1} z^{q^{*} i}+\text { h.o.t. }
$$

Considering the uniqueness in Lemma 2.5 and Theorem 3.1, from expressions (3.23) and (3.24), it is easy to get expression (3.21) and $\tilde{\varphi}=\tilde{f}, \tilde{\psi}=\tilde{g}$ with mathematical induction.

Theorems 3.1 and 3.2 give a new algorithm to compute the generalized period constants at the origin of system (3.2) by applying the coefficients of system (1.11). For any a positive integer $l$, in order to compute $\tau_{l}$, we only need to force addition, subtraction, multiplication, and division to the coefficients of system (1.11). The algorithm is recursive and then avoids complex integration operations and solving equations. It is symbolic and easy to realize with computer algebraic system such as Mathematica or Maple. 
Remark 3.3. We cannot use Theorems 3.1 and 3.2 to compute singular point quantities $\mu_{l}=$ $p_{l}-q_{l}(l=1,2, \ldots)$, because the one condition of Theorem 3.2 is expression (3.20), while the computation of $\mu_{l}$ is only under the condition $\mu_{0}=\mu_{1}=\cdots=\mu_{l-1}=0$. However, we can apply directly the two theorems to find necessary conditions for linearizability, needless to solve firstly the problem of integrability.

\section{Application}

In this section, we investigate the linearizability problem at degenerate singular point for the following septic system:

$$
\begin{gathered}
\frac{d z}{d T}=z^{2} w+b_{03} z^{5}+b_{12} z^{4} w+b_{21} z^{3} w^{2}+b_{30} z^{2} w^{3}+\lambda z^{4} w^{3} \\
\frac{d w}{d T}=-w^{2} z-a_{03} w^{5}-a_{12} w^{4} z-a_{21} w^{3} z^{2}-a_{30} w^{2} z^{3}-\lambda w^{4} z^{3}
\end{gathered}
$$

where

$$
b_{k j}=\overline{a_{k j}}, \quad(k, j) \in\{(3,0),(2,1),(1,2),(0,3)\} .
$$

Factually, the pseudo-isochronous center problem at the degenerate singular point of system $(4.1)$ has already been solved in $[17,18]$. Here the aim that we chose this system as an illustrative example is just to demonstrate the effectiveness of this theory.

Firstly, let us see the computational method of singular point quantities at 1:-1 resonant degenerate singular point.

When $p=q=1$, by the time scaling $T \rightarrow 1 /(2 n+3) T$, system (3.2) can be simplified as

$$
\begin{gathered}
\frac{d z}{d T}=z+\frac{1}{2 n+3} \sum_{\alpha+\beta=2 n+2}^{\infty}\left[(n+2) a_{\alpha \beta}+(n+1) b_{\beta+1, \alpha-1}\right] z^{\alpha+1} w^{\beta+1} \\
\times(z w)^{(\alpha+\beta-2 n-2)(n+1)}=Z(z, w), \\
\frac{d w}{d T}=-w-\frac{1}{2 n+3} \sum_{\alpha+\beta=2 n+2}^{\infty}\left[(n+2) b_{\alpha \beta}+(n+1) a_{\beta+1, \alpha-1}\right] w^{\alpha+1} z^{\beta+1} \\
\times(z w)^{(\alpha+\beta-2 n-2)(n+1)}=-W(z, w) .
\end{gathered}
$$

Definition 4.1 (see [19]). (i) For any positive integer $k, \mu_{k}=(2 n+3) \mu_{(2 n+3) k}^{\prime}$ is called the $k$ th singular point quantity at the origin of system $(1.12)$, where $\mu_{(2 n+3) k}^{\prime}$ is the $(2 n+3) k$ th singular point quantity at the origin of system (4.3).

(ii) If $\mu_{0}=\mu_{1}=\cdots=\mu_{k-1}=0, \mu_{k} \neq 0$, the origin is called the $k$ th fine singular point of system (1.12).

(iii) If for all $k, \mu_{k}=0$, then the origin is called a complex center of system (1.12).

Definition 4.2. Degenerate singular point of system (1.12) or the origin of system (3.2) $p=q=1$ is called a complex pseudo-isochronous center if the origin of system (4.3) is a complex isochronous center. 
Journal of Applied Mathematics

Lemma 4.3 (see [19]). For system (4.3), one can derive successively the terms of the following formal series:

$$
M(z, w)=1+\sum_{k=1}^{\infty} f_{(2 n+3) k}(z, w)
$$

where

$$
f_{(2 n+3) k}(z, w)=\sum_{\alpha+\beta=(2 n+3) k} c_{\alpha \beta} z^{\alpha} w^{\beta}
$$

such that

$$
\begin{aligned}
\frac{\partial M}{\partial z} Z-\frac{\partial M}{\partial w} W+\left(\frac{\partial Z}{\partial z}-\frac{\partial W}{\partial w}\right) M & =\sum_{m=1}^{\infty} \frac{2 m n+3 m+1}{2 n+3} \lambda_{m}(z w)^{(2 n+3) m+1}, \\
\lambda_{m} & \sim \mu_{m}, \quad m=1,2, \ldots
\end{aligned}
$$

Lemma 4.4 (see [19]). For all pairs $(\alpha, \beta)$ with $\alpha \neq \beta$, when $\alpha+\beta=(2 n+3) N, c_{\alpha \beta}$ is given by

$$
\begin{aligned}
c_{\alpha \beta}=\frac{1}{(2 n+3)(\beta-\alpha)} \sum_{k+j=2 n+3}^{2 n+2+N}\{ & {[(n+2) \alpha-(n+1) \beta+1] a_{k, j-1} } \\
& \left.-[(n+2) \beta-(n+1) \alpha+1] b_{j, k-1}\right\} \\
\times & c_{\alpha-(n+2) k-(n+1) j+(n+1)(2 n+3), \beta-(n+2) j-(n+1) k+(n+1)(2 n+3),}
\end{aligned}
$$

where, in (4.5), one take $c_{00}=1$ and $c_{k k}$ as an arbitrary constant $(k=1,2, \ldots)$.

For any positive integer $m, \lambda_{m}$ is given by

$$
\lambda_{m}=\sum_{k+j=1}^{2 n+2+2 m}\left(a_{k, j-1}-b_{j, k-1}\right) \mathcal{c}_{(m+n+1)(2 n+3)-(n+2) k-(n+1) j,(m+n+1)(2 n+3)-(n+2) j-(n+1) k,}
$$

where for all pairs $(\alpha, \beta)$, when $\alpha<0$ or $\beta<0$, one take $a_{\alpha \beta}=b_{\alpha \beta}=c_{\alpha \beta}=0$.

Performing the transformation (3.1) $)_{n=1}$ and renaming $\left(z_{1}, w_{1}, T_{1}\right)$ by $(z, w, T)$, system (4.1) is reduced to

$$
\begin{aligned}
\frac{d z}{d T}= & 5 z+3 b_{03} z^{8} w^{3}+\left(2 a_{30}+3 b_{12}\right) z^{7} w^{4}+\left(2 a_{21}+3 b_{21}\right) z^{6} w^{5}+\left(2 a_{12}+3 b_{30}\right) z^{5} w^{6} \\
& +2 a_{03} z^{4} w^{7}+5 \lambda z^{11} w^{10}, \\
\frac{d w}{d T}= & -5 w-3 a_{03} w^{8} z^{3}-\left(2 b_{30}+3 a_{12}\right) w^{7} z^{4}-\left(2 b_{21}+3 a_{21}\right) w^{6} z^{5}-\left(2 b_{12}+3 a_{30}\right) w^{5} z^{6} \\
& -2 b_{03} w^{4} z^{7}-5 \lambda w^{11} z^{10} .
\end{aligned}
$$


By the time scaling $T \rightarrow(1 / 5) T$, system (4.9) can be interpreted to

$$
\begin{aligned}
\frac{d z}{d T}= & z+\frac{3}{5} b_{03} z^{8} w^{3}+\frac{1}{5}\left(2 a_{30}+3 b_{12}\right) z^{7} w^{4}+\frac{1}{5}\left(2 a_{21}+3 b_{21}\right) z^{6} w^{5}+\frac{1}{5}\left(2 a_{12}+3 b_{30}\right) z^{5} w^{6} \\
& +\frac{2}{5} a_{03} z^{4} w^{7}+\lambda z^{11} w^{10} \\
\frac{d w}{d T}= & -w-\frac{3}{5} a_{03} w^{8} z^{3}-\frac{1}{5}\left(2 b_{30}+3 a_{12}\right) w^{7} z^{4}-\frac{1}{5}\left(2 b_{21}+3 a_{21}\right) w^{6} z^{5}-\frac{1}{5}\left(2 b_{12}+3 a_{30}\right) w^{5} z^{6} \\
& -\frac{2}{5} b_{03} w^{4} z^{7}-\lambda w^{11} z^{10} .
\end{aligned}
$$

According to Definition 4.1, Lemmas 4.3 and 4.4, we have the following.

Theorem 4.5. The first 9 singular point quantities at degenerate singular point of system (4.1) are as follows:

$$
\begin{aligned}
\mu_{1}= & -a_{21}+b_{21}, \\
\mu_{2}= & a_{12} a_{30}-b_{12} b_{30}, \\
\mu_{3}= & \frac{1}{8}\left(-9 a_{03} a_{30}^{2}-a_{12}^{2} b_{03}+a_{03} b_{12}^{2}+9 b_{03} b_{30}^{2}\right), \\
\mu_{4}= & -\frac{1}{12}\left(a_{21}+b_{21}\right)\left(a_{12}^{2} b_{03}+3 a_{03} a_{30} b_{12}-a_{03} b_{12}^{2}-3 a_{12} b_{03} b_{30}\right), \\
\mu_{5}= & -\frac{1}{648}\left(a_{12}^{2} b_{03}+3 a_{03} a_{30} b_{12}-a_{03} b_{12}^{2}-3 a_{12} b_{03} b_{30}\right)\left(-27 a_{03} b_{03}+16 a_{12} b_{12}+216 \lambda\right), \\
\mu_{6}= & 0, \\
\mu_{7}= & -\frac{1}{466560}\left(405 a_{03}^{2} b_{03}^{2}-8928 a_{03} a_{12} b_{03} b_{12}+6400 a_{12}^{2} b_{12}^{2}\right) \\
& \times\left(-a_{12}^{2} b_{03}-3 a_{03} a_{30} b_{12}+a_{03} b_{12}^{2}+3 a_{12} b_{03} b_{30}\right), \\
\mu_{8}= & \frac{7}{69984}\left(45 a_{03} b_{03}-32 a_{12} b_{12}\right)\left(a_{12}^{2} b_{03}+a_{03} b_{12}^{2}\right)\left(-a_{12}^{2} b_{03}-3 a_{03} a_{30} b_{12}+a_{03} b_{12}^{2}+3 a_{12} b_{03} b_{30}\right), \\
\mu_{9}= & \frac{11}{13778100} a_{12}^{2} b_{12}^{2}\left(-224181 a_{03} b_{03}+164000 a_{12} b_{12}\right)\left(a_{12}^{2} b_{03}+3 a_{03} a_{30} b_{12}-a_{03} b_{12}^{2}-3 a_{12} b_{03} b_{30}\right) .
\end{aligned}
$$

In the above expression of $\mu_{k}$, one has already let $\mu_{1}=\cdots=\mu_{k-1}=0, k=2,3, \ldots, 9$.

From Theorem 4.5, we get the following.

Theorem 4.6. For system (4.1), the first 9 singular point quantities are zero if and only if one of the following conditions holds:

(I) $a_{21}=b_{21}, 3 a_{30}-b_{12}=0,3 b_{30}-a_{12}=0, a_{12} b_{12} \neq 0$;

(II) $a_{21}=b_{21}, a_{30} a_{12}=b_{30} b_{12}, a_{12}^{2} b_{03}=b_{12}^{2} a_{03}, b_{30} a_{12} b_{03}=a_{30} b_{12} a_{03}, b_{30}^{2} b_{03}=a_{30}^{2} a_{03}$. 
In order to obtain the center conditions of degenerate singular point, we have to find out all the elementary Lie invariants of system (4.1); following the technique used in [10], we have the following.

Lemma 4.7. All the elementary Lie invariants of system (4.1) are as follows:

$$
\begin{array}{ll}
\lambda, a_{21}, b_{21}, a_{30} b_{30}, a_{12} b_{12}, a_{03} b_{03}, a_{30} a_{12}, & b_{30} b_{12} \\
a_{30}^{2} a_{03}, & a_{30} b_{12} a_{03}, b_{12}^{2} a_{03}, b_{30}^{2} b_{03}, b_{30} a_{12} b_{03}, a_{12}^{2} b_{03} .
\end{array}
$$

Theorem 4.8. For system (4.1), all the singular point quantities at degenerate singular point are zero if and only if the first 9 singular point quantities are zero, that is, one of the conditions in Theorem 4.6 holds. Correspondingly, the conditions in Theorem 4.6 are the center conditions of degenerate singular point.

Proof. If condition (I) is satisfied, system (4.1) has the integrating factor $(z w)^{-5}$; if condition (II) is satisfied, system (4.1) satisfies the conditions of the extended symmetric principle (Theorem 2.6 in [10]).

We next discuss the linearizability problem of the origin of system (4.9), according to Theorems 3.1 and 3.2; we can get the recursive formulae to compute period constants at the origin. Since transformation $(3.1)_{n=1}$ is a homeomorphism, the center conditions of the origin of system (4.9) are identical with those of degenerate singular point of system (4.1). From the center conditions in Theorem 4.6, we investigate the following two cases.

Case 1 (center condition (I) holds). Substituting center condition (I) into the recursive formulae in Theorem 3.1 we obtain the first 8 period constants

$$
\begin{aligned}
& \tau_{1}=2 r_{21}, \\
& \tau_{2}=\frac{1}{18}\left(-9 a_{03} b_{03}-16 a_{12} b_{12}+36 \lambda\right), \\
& \tau_{3}=0, \\
& \tau_{4}=\frac{1}{2592}\left(-81 a_{03}^{2} b_{03}^{2}+1152 a_{03} a_{12} b_{03} b_{12}-256 a_{12}^{2} b_{12}^{2}\right), \\
& \tau_{5}=\frac{7}{1944}\left(-27 a_{03} b_{03}+32 a_{12} b_{12}\right)\left(a_{12}^{2} b_{03}+a_{03} b_{12}^{2}\right), \\
& \tau_{6}=0, \\
& \tau_{7}=\frac{14228}{10935} a_{12}^{2} b_{12}^{2}\left(a_{12}^{2} b_{03}+a_{03} b_{12}^{2}\right), \\
& \tau_{8}=-\frac{65}{59049} a_{12}^{3} b_{12}^{3}\left(-5571 a_{03} b_{03}+1280 a_{12} b_{12}\right) .
\end{aligned}
$$

In the above expression of $\tau_{k}$, we have already let $\tau_{1}=\cdots=\tau_{k-1}=0, k=2,3, \ldots, 8$. From $\tau_{7}=0$, there exists a complex constant $h$, such that

$$
a_{03}=h a_{12}^{2}, \quad b_{03}=-h b_{12}^{2},
$$


putting (4.14) into the expression of $\tau_{4}$, we have

$$
\tau_{4}=-\frac{1}{2592} a_{12}^{2} b_{12}^{2}\left(256+1152 a_{12} b_{12} h^{2}+81 a_{12}^{2} b_{12}^{2} h^{4}\right) .
$$

By virtue of $a_{12} b_{12} \neq 0, \tau_{4}=0$ yields

$$
h=\frac{4}{3} \sqrt{\frac{-4 \pm \sqrt{15}}{a_{12} b_{12}}},
$$

this, together with (4.14), leads to

$$
\tau_{8}=\frac{1040}{59049}(2396 \mp 619 \sqrt{15}) a_{12}^{4} b_{12}^{4} \neq 0 .
$$

Therefore, under center condition (I), the origin is not a complex isochronous center.

Case 2 (center condition (II) holds). When center condition (II) holds, the right hand of system (4.9) satisfies the extended symmetric principle (Theorem 2.6 in [10]). Write that $3 a_{30}-$ $b_{12}=B, 3 b_{30}-a_{12}=A$. Then when $A=B=0$, center condition (I) holds. We next assume that $A B \neq 0$. Hence, we have from center condition (II) that

$$
a_{30} A-b_{30} B=0, \quad a_{03} B^{2}-b_{03} A^{2}=0 .
$$

It follows that there exist $r_{21}, r, s$ such that

$$
a_{21}=b_{21}=r_{21}, \quad a_{30}=r B, \quad b_{30}=r A, \quad a_{03}=s A^{2}, \quad b_{03}=s B^{2},
$$

then center condition (II) is equivalent to

$$
\begin{aligned}
& a_{21}=b_{21}=r_{21}, \quad a_{30}=r B, \quad b_{30}=r A, \quad a_{12}=(3 r-1) A, \quad b_{12}=(3 r-1) B, \\
& a_{03}=s A^{2}, \quad b_{03}=s B^{2} .
\end{aligned}
$$

System (4.9) is brought to

$$
\begin{gathered}
\frac{d z}{d T}=5 z+3 B^{2} s z^{8} w^{3}+B(11 r-3) z^{7} w^{4}+5 r_{21} z^{6} w^{5}+A(9 r-2) z^{5} w^{6}+2 A^{2} s z^{4} w^{7}+5 \lambda z^{11} w^{10}, \\
\frac{d w}{d T}=-5 w-3 A^{2} s w^{8} z^{3}-A(11 r-3) w^{7} z^{4}-5 r_{21} w^{6} z^{5}-B(9 r-2) w^{5} z^{6}-2 B^{2} s w^{4} z^{7}-5 \lambda w^{11} z^{10}
\end{gathered}
$$

Putting expression (4.20) into the recursive formulae in Theorem 3.1 and calculating in Mathematica, we have the following. 
Theorem 4.9. The first 6 period constants of the origin of system (4.21) are given by

$$
\begin{aligned}
& \tau_{1}=2 r_{21} \\
& \tau_{2}=\frac{1}{2}\left(4 A B r-16 A B r^{2}-A^{2} B^{2} s^{2}+4 \lambda\right) \\
& \tau_{3}=\frac{1}{4} A^{2} B^{2}(-1+6 r) s \\
& \tau_{4}=-2 A^{2} B^{2}(-1+r) r^{2}(-1+4 r)-\frac{1}{288} A^{3} B^{3} s^{2}\left(-16+9 A B s^{2}\right), \\
& \tau_{5}=-\frac{1}{972} A^{3} B^{3} r\left(-8+27 A B s^{2}\right) \\
& \tau_{6}=\frac{1}{25} A^{3} B^{3} r^{2}(-1+4 r)\left(-154+715 r-1067 r^{2}+531 r^{3}\right)-\frac{5053}{77760} A^{4} B^{4} s^{2} .
\end{aligned}
$$

In the above expression of $\tau_{k}$, one has already let $\tau_{1}=\cdots=\tau_{k-1}=0, k=2,3, \ldots, 6$.

Theorem 4.10. The first 6 period constants at the origin of system (4.21) are zero if and only if one of the following conditions holds:

$$
\begin{gathered}
\lambda=r_{21}=r=s=0, \quad A B \neq 0, \\
\lambda=r_{21}=s=0, \quad r=\frac{1}{4}, A B \neq 0 .
\end{gathered}
$$

Proof. Being $A B \neq 0, \tau_{3}=0$ implies $r=1 / 6$ or $s=0$.

When $r=1 / 6, \tau_{4}=-(1 / 2592) A^{2} B^{2}\left(40-144 A B s^{2}+81 A^{2} B^{2} s^{4}\right)$. From $\tau_{4}=0$, we have $s=$ $\pm(1 / 3) \sqrt{2(4 \pm \sqrt{ } 6) / A B}$, then $\tau_{5}=(\mp 1 / 729) \sqrt{(1 / 2)(4 \pm \sqrt{6})}( \pm 8+3 \sqrt{6}) \sqrt{A^{5} B^{5}} \neq 0$. When $s=0, \tau_{4}=-2 A^{2} B^{2}(-1+r) r^{2}(-1+4 r), \tau_{6}=(1 / 25) A^{3} B^{3} r^{2}(-1+4 r)\left(-154+715 r-1067 r^{2}+\right.$ $\left.531 r^{3}\right)$. From $\tau_{4}=\tau_{6}=0$, we get $r=0$ or $r=1 / 4$.

Theorem 4.11. Under center condition (II), the origin of system (4.21) is a complex pseudo-isochronous center if and only if one of the conditions in Theorem 4.10 holds.

Proof. When condition (4.23) is satisfied, after the time scaling $T \rightarrow(1 / 5) T$, system (4.21) becomes

$$
\begin{gathered}
\frac{d z}{d T}=\frac{1}{5} z\left(5-3 B z^{6} w^{4}-2 A z^{4} w^{6}\right) \\
\frac{d w}{d T}=-\frac{1}{5} w\left(5-3 A w^{6} z^{4}-2 B w^{4} z^{6}\right) .
\end{gathered}
$$

There exists a transformation

$$
u=\frac{z\left(1-A z^{4} w^{6}\right)^{1 / 5}}{\left(1-B z^{6} w^{4}\right)^{3 / 10}}, \quad v=\frac{w\left(1-B w^{4} z^{6}\right)^{1 / 5}}{\left(1-A w^{6} z^{4}\right)^{3 / 10}}
$$

such that system (4.25) is reduced to $d u / d T=u, d v / d T=-v$. 
When condition (4.24) is satisfied, after the time scaling $T \rightarrow(1 / 5) T$, system (4.21) becomes

$$
\begin{gathered}
\frac{d z}{d T}=\frac{1}{20} z\left(20-B z^{6} w^{4}+A z^{4} w^{6}\right), \\
\frac{d w}{d T}=-\frac{1}{20} w\left(20-A w^{6} z^{4}+B w^{4} z^{6}\right),
\end{gathered}
$$

from the polar coordinate transformation

$$
z=\rho e^{i \theta}, \quad w=\rho e^{-i \theta}, \quad T=i t, \quad i=\sqrt{-1},
$$

and we have for system (4.27) that

$$
\frac{d \theta}{d t}=\frac{1}{2}\left(\frac{1}{z} \frac{d z}{d T}-\frac{1}{w} \frac{d w}{d T}\right)=1
$$

From the above discussion and Definition 4.2, the conclusion of this theorem follows.

We have from Definition 4.2, expression (4.20), Theorems 4.10 and 4.11 the following.

Theorem 4.12. Degenerate singular point of system (4.1) is a complex pseudo-isochronous center if and only if one of the following conditions is satisfied:

(i) $\lambda=a_{30}=b_{30}=a_{21}=b_{21}=a_{03}=b_{03}=0, \quad a_{12} b_{12} \neq 0$,

(ii) $\lambda=a_{21}=b_{21}=a_{03}=b_{03}=0, a_{30}+b_{12}=0, b_{30}+a_{12}=0, a_{12} b_{12} \neq 0$.

\section{Conclusions and Remarks}

In the qualitative theory of ODE, the linearizability (or isochronicity) problem is one of the open problems for polynomial differential systems which is far to be solved in general. Up to now, most of the existing literatures are concerned with elementary singular point. A progressive way to find necessary conditions for linearizability is to compute period constants.

The linearizability problem of degenerate singular point is much more difficult. As far as the degenerate case is concerned, the classical methods are invalid. As a result, the literatures about linearizability problem of degenerate singular point are limited. One reason is that the problem itself is very complicated; another is that the problem is subject to limited methods. Therefore, it is completely natural to develop a "new" theory to analyze this dynamical behavior for the planar dynamical systems with degenerate singular points.

In our paper, firstly, the degenerate singular point is taken to the elementary origin by a blow-up; besides, we establish a new recursive algorithm to compute the so-called generalized period constants, so we can deal with the linearizability problem of degenerate singular point via the classical theory developed for elementary singular point. Synthesizing the above, we give an indirect approach regarding to the linearizability problem of the degenerate singular point. 


\section{Acknowledgments}

The authors are grateful to the anonymous referees for their careful comments and valuable suggestions. Especially, the authors greatly appreciated the help of the referees in posing important questions, which are sure to enhance the readability and interest of the paper and suggest some neat ideas for future studies. This work is supported in part by the National Nature Science Foundation of China (NSFC 11101126 and 11101127) and Scientific Research Foundation for Doctoral Scholars of HAUST (09001524).

\section{References}

[1] Y. P. Lin and J. B. Li, "The normal form of a planar autonomous system and critical points of the period of closed orbits," Acta Mathematica Sinica, vol. 34, no. 4, pp. 490-501, 1991 (Chinese).

[2] W. S. Loud, "Behavior of the period of solutions of certain plane autonomous systems near centers," Contributions to Differential Equations, vol. 3, pp. 21-36, 1964.

[3] J. Chavarriga, J. Giné, and I. A. García, "Isochronous centers of a linear center perturbed by fourth degree homogeneous polynomial," Bulletin des Sciences Mathématiques, vol. 123, no. 2, pp. 77-96, 1999.

[4] J. Chavarriga, J. Giné, and I. A. García, "Isochronous centers of a linear center perturbed by fifth degree homogeneous polynomials," Journal of Computational and Applied Mathematics, vol. 126, no. $1-2$, pp. 351-368, 2000.

[5] I. Pleshkan, "A new method of investigating the isochronicity of a system of two differential equations," Differential Equations, vol. 5, pp. 796-802, 1969.

[6] L. Cairó, J. Chavarriga, J. Giné, and J. Llibre, "A class of reversible cubic systems with an isochronous center," Computers \& Mathematics with Applications, vol. 38, no. 11-12, pp. 39-53, 1999.

[7] J. Chavarriga, I. A. García, and J. Giné, "Isochronicity into a family of time-reversible cubic vector fields," Applied Mathematics and Computation, vol. 121, no. 2-3, pp. 129-145, 2001.

[8] J. Chavarriga, J. Giné, and I. García, "Isochronous centers of cubic systems with degenerate infinity," Differential Equations and Dynamical Systems, vol. 7, no. 2, pp. 221-238, 1999.

[9] N. G. Lloyd, C. J. Christopher, J. Devlin, J. M. Pearson, and N. Yasmin, "Quadratic-like cubic systems," Differential Equations and Dynamical Systems, vol. 5, no. 3-4, pp. 329-345, 1997.

[10] Y. Liu and J. Li, "Theory of values of singular point in complex autonomous differential system," Science in China (Series A), vol. 3, pp. 10-24, 1990.

[11] Y. Liu and W. Huang, "A new method to determine isochronous center conditions for polynomial differential systems," Bulletin des Sciences Mathématiques, vol. 127, no. 2, pp. 133-148, 2003.

[12] C. Christopher, P. Mardešić, and C. Rousseau, "Normalizable, integrable, and linearizable saddle points for complex quadratic systems in $\mathbb{C}^{2}, "$ Journal of Dynamical and Control Systems, vol. 9, no. 3, pp. 311-363, 2003.

[13] S. Gravel and P. Thibault, "Integrability and linearizability of the Lotka-Volterra system with a saddle point with rational hyperbolicity ratio," Journal of Differential Equations, vol. 184, no. 1, pp. 20-47, 2002.

[14] C. Liu, G. Chen, and C. Li, "Integrability and linearizability of the Lotka-Volterra systems," Journal of Differential Equations, vol. 198, no. 2, pp. 301-320, 2004.

[15] Q. Wang and Y. Liu, "Linearizability of the polynomial differential systems with a resonant singular point," Bulletin des Sciences Mathématiques, vol. 132, no. 2, pp. 97-111, 2008.

[16] V. V. Amelkin, N. A. Lukashevich, and A. P. Sadovskii, Nonlinear Oscillations in Second Order Systems, BSU, Minsk, Russia, 1982.

[17] Y. Wu and C. Zhang, "Bifurcation of limit cycles and pseudo-isochronicity at degenerate singular point in a septic system," Results in Mathematics, vol. 57, no. 1-2, pp. 97-119, 2010.

[18] Y. Wu, C. Zhang, and P. Li, "Isochronicity problem of higher-order singular point for polynomial differential systems," Acta Applicandae Mathematicae, vol. 110, no. 3, pp. 1429-1448, 2010.

[19] Y. Liu, J. Li, and W. Huang, Singular Point Values, Center Problem and Bifurcation of Limit Cycles of Two Dimensional Differential Autonomous Systems, Science Press, Beijing, China, 2008. 


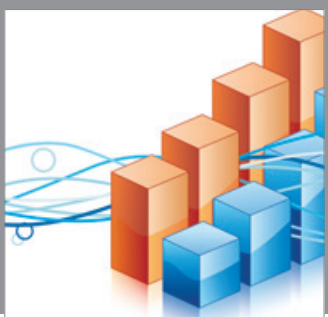

Advances in

Operations Research

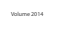

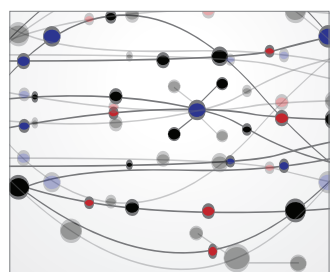

\section{The Scientific} World Journal
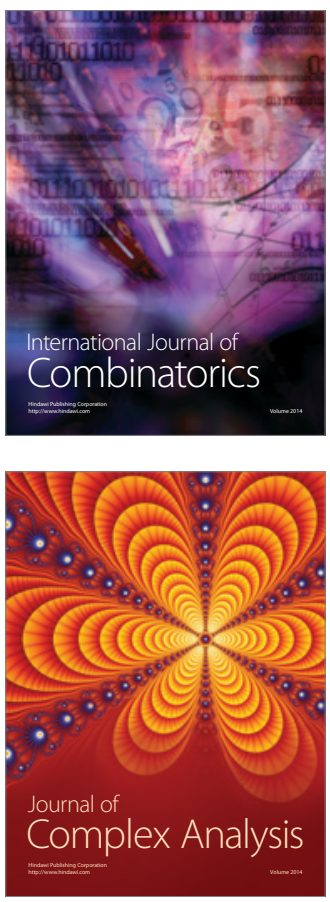

International Journal of

Mathematics and

Mathematical

Sciences
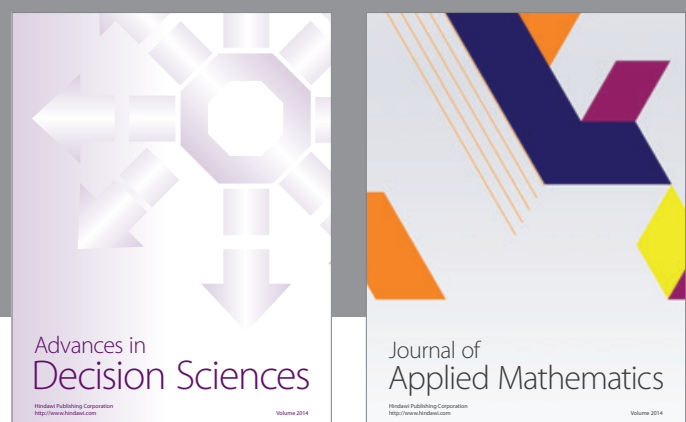

Journal of

Applied Mathematics
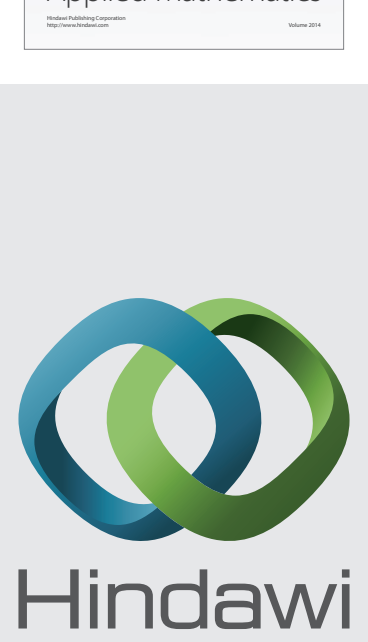

Submit your manuscripts at http://www.hindawi.com
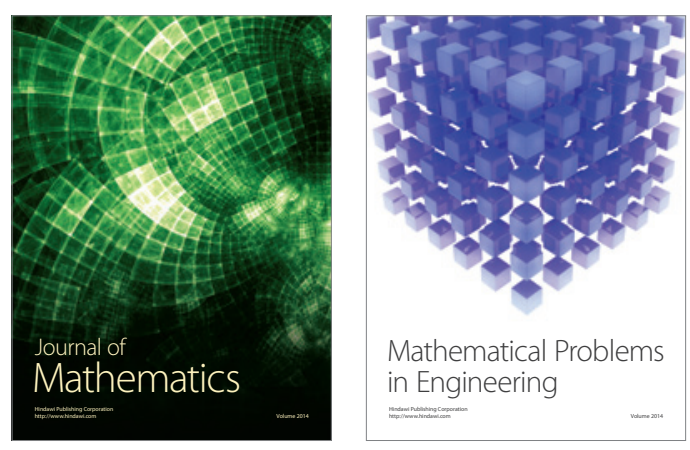

Mathematical Problems in Engineering
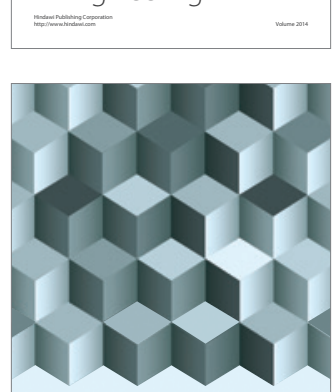

Journal of

Function Spaces
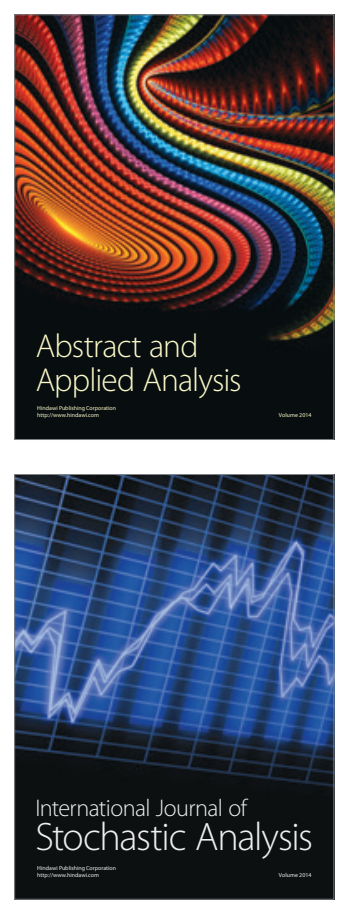

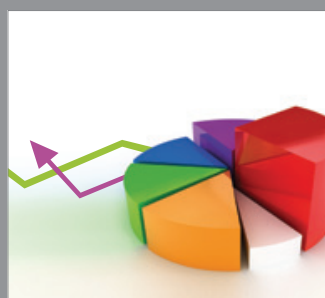

ournal of

Probability and Statistics

Promensencen
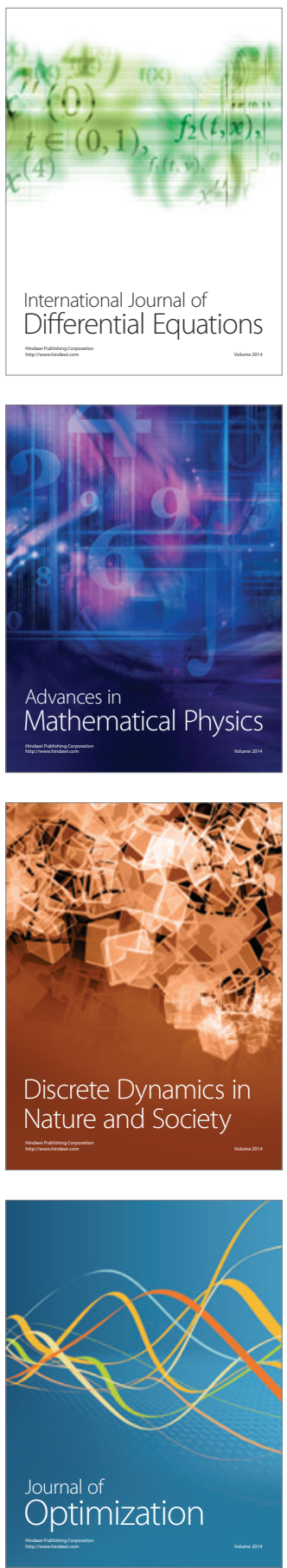